\title{
Relay Based Performance Metrics of Bluetooth Scatternet Scheduling
}

\author{
K.R. Kanagavalli, B. Sankaragomathi \\ Deartment of Computer Science, \\ Government Arts and Science College, Sivakasi, Tamilnadu, India
}

\begin{abstract}
Article Info
Article history:

Received Jan 31, 2018

Revised Apr 21, 2018

Accepted Apr 30, 2018

Keywords:

Bluetooth

Piconet

Scatternet

Relay Node

Scheduling

ABSTRACT

Bluetooth is an emerging mobile ad-hoc network that accredits wireless communication to connect various short range devices. A single hop network called piconet is the basic communication topology of bluetooth which allows only eight active devices for communication among them seven are active slaves controlled by one master. Multiple piconets are interconnected through a common node, known as Relay, to form a massive network called as Scatternet. It is obvious that the performance of Scatternet scheduling is highly dependent and directly proportionate with the performance of the Relay node. In contrary, by reducing the number of Relays, it may lead to poor performance, since every Relay has to perform and support several piconet connections. The primary focus of this study is to observe the performance metrics that affects the inter-piconet scheduling since the Relay node's role is like switch between multiple piconets. In this paper, we address and analyze the performance issues to be taken into consideration for efficient data flow in Scatternet based on Relay node.
\end{abstract}

Copyright (c) 2018 Institute of Advanced Engineering and Science. All rights reserved.

\section{Corresponding Author:}

K.R. Kanagavalli, Deartment of Computer Science, Government Arts and Science College, Sivakasi, Tamilnadu, India.

Email: krkanaga_valli@yahoo.com

\section{INTRODUCTION}

Bluetooth is a modern low power wireless technology which provides favorable communication for mobile devices such as cellular phones, palmtop, laptops and any electronic device which are all portable. Due to the demand for networking between these devices, Bluetooth is a promising proposed standard for shortrange, low power wireless communication. It is the best feasible communication for short range mobile ad hoc networking in addition to the cable replacement technology [1, 2].

The Bluetooth system operates with Industrial-Scientific-Medical (ISM) $2.4 \mathrm{GHz}$ frequency band with the worldwide license free bandwidth of $1 \mathrm{Mbps}$. Frequency Hopping (FH) technique is used to avoid interference, in which at every packet transmission the carrier frequency is changed. For Bluetooth defined hop carriers are 79 with the spacing of $1 \mathrm{MHz}$ in ISM band.

Bluetooth devices must form networks before the actual communication can start [1]. In simplest form, the Bluetooth specification will allow only eight active nodes or devices to communicate within its smallest and centralized networking unit known as piconet. Among them one device has the role of master which controls the access to the channel, while the other seven are slaves. All the communications take place under master's control. There should be only one master that controls the channel access within the piconet. At any given instance 7 slaves are active, and up to 255 others can be parked with the condition that they are still noticing to the communication in a piconet [1]. Every piconet master regulates a frequency hopping in sequence, which the slaves should follow for being synchronized with the piconet channel. The master and slaves are communicating between them using the Time Division Duplex (TDD) slots. The channel is being 
divided into $625 \mu$ s slots. Both synchronous and asynchronous connection can be setup in piconet between master and slave.

The master-to-slave transmission starts in even numbered slots, while slave-to-master transmission starts in odd numbered slots. The communication from Master to slave is referred as downlink and the communication from the slave to master is referred as uplink. A downlink packet with the subsequent uplink packet is referred as Bluetooth frame.

The slaves polled by the master by sending packets with appropriate identification and data. The slaves can response to the master only when addressed by the master. Data are transmitted as packets which take 1, 3 or 5-slots, in consecutive slots. The frequency remains unchanged during the transmission of packet; ahead the transmission of next packet the frequency is altered which is called fast hopping and enhances the communication reliability. When the master has no data for the slave, it sends zero payload 1-slot POLL packet to the slave and in response 1-slot NULL packet sends by the slave. The master and the addressed slave use the same communication channel, not at the same time [1, 3].

If many bluetooth devices exist in a same area and exceed the limit of piconet, a larger network will be formed that has multiple piconet known as Scatternet. A Bluetooth device can participate in more than one piconet. A device that is a member of more than one piconet is referred as bridge or relay node. There is certain restriction on scatternet scheduling in the presence of bridge nodes and other slaves [3 - 7].

At any time, a relay can active in only one piconet. Therefore, the communication with other piconets' is blocked. When the relay has finished its service for the particular time in the current piconet; it switches to the next piconet. It causes delay and decreases the performance of the network. This issue is known as an inter-piconet scheduling problem. There have been many protocols developed for inter-piconet communications.

The source and destination devices may be in different piconets need many hops to transfer the data, because scatternet is a multi-hop communication network. A number of research papers proposed for routing packets from source to destination. Considerable amount of work has been done in the area of bridge switching among piconets that is inter-piconet scheduling. An inefficient inter-piconet scheduling may degrade the system performance. However, the Bluetooth specification does not specifically prescribe any inter-piconet scheduling scheme. The solutions of the problems are left open to researchers. Therefore, different techniques have been developed for inter-piconet communication [8,9].

The aim of the paper is to evaluate the performance metrics of relay based inter-piconet scheduling and to give some insight into the problem of designing efficient and practical schemes. Analytic performance evaluation of inter-piconet scheduling algorithms has great importance, since it may provide insight on their design and optimization.

This paper is organized as follows. Section 2 gives brief introduction to the Bluetooth scatternet technology while section 3 presents performance metrics. In section 4 , we analyse the simulation results. We summarize the work in section 5 .

\section{BACKGROUND}

A piconet may contain a maximum of eight devices. If the number of devices exceeds eight, then to cover all devices at least two piconets are required. Scatternet is formed by interconnecting several piconets. Any device in the network can play three different roles master, slave or relay within piconet. A device that coordinates the function within the piconet and has the complete knowledge of piconet is called Master. Slaves are communicated only through the master. Relay node connects the piconets to form the scatternet. Through the relay node only packets within the scatternet are transmitted from one piconet to another.

Scatternet formed by interconnecting two piconets P1 and P2 is shown in the Figure 1 . The term $S=$ $\{P 1, P 2\}$ denotes the scatternet where $\mathrm{P} 1$ and $\mathrm{P} 2$ are piconets. Both piconets have one master each and four and five slaves respectively. Piconet P1 has 4 devices, one is master, 3 slaves out of this 2 nodes are relay nodes denoted by $P 1=\{m 1, s 11, r 1, r 2\}$. Piconet $\mathrm{P} 2$ has five nodes with one master and 3 slave nodes and 2 relay nodes, $P 2=\{m 2, s 21, s 22, s 23, r 1, r 2\}$. Relay nodes $r 1$ and $r 2$ are participating in two piconets simultaneously, $R=\{r 1, r 2\}$. One possible route path for data transmission from slave node(s11) in the piconet P1 to slave node(s22) in piconet P2 is denoted as $\mathrm{s} 11 \rightarrow \mathrm{m} 1 \rightarrow \mathrm{r} 1 \rightarrow \mathrm{m} 2 \rightarrow \mathrm{s} 22$.

Master in each piconet assigns a unique Active Member Address (AM_Addr) to each active slave. As mentioned above, Bluetooth follows Time-Division-Duplex access scheme to divide 1 second into 1600 time slots of $625 \mu \mathrm{s}$. Under the control of master, the even time slot is reserved for master to transmit packet to its slave. Slave that receives packet from master in even slot has the right to transmit packet to master in the next odd slot. A relay that simultaneously participates in multiple piconets switches among piconets and synchronizes with the hopping sequence of each piconet to which it switches. 


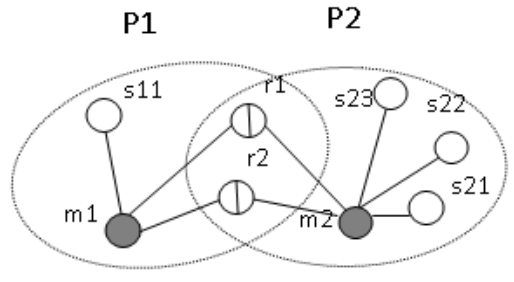

Figure 1. Scatternet Structure

The Bluetooth technology standard [1] has not yet specified the way how the bridges should schedule their visits in different piconets. This is currently a subject of intense research effort. There are some issues that make scatternet application unreliable, even though the functionality of scatternet works well. First the bridge unit can change the role as either master or slave Second, an issue that the Bluetooth specification does not dealing with when multiple profiles are being run simultaneously on one device. The quality of a scatternet can be measured by many performance metrics.

A master controls the flow of packets between the slaves of its piconet. It consumes higher energy. Hence, it is preferred to minimize the number of masters in a scatternet.Similarly, the number of bridges shall be minimized because a bridge node schedules its tasks between the piconets it belongs to, and thus, bridge may consume more energy. More specifically, an M/S bridge may cause degradation in the throughput of its piconet, as all activities in its piconets are postponed when that bridge serves as a slave.

\section{PERFORMANCE METRICS}

The primary metrics we have taken into consideration to evaluate performance of the scheduling are Link Breakage Recovery, Link Repairing, Link Breakage Prediction and Relay Reduction. The description of the parameters is briefed:

\subsection{Link Breakage Recovery}

The relay nurtures connection between different piconets. The mobility of the relay disconnects scatternets which is defined as Link breakage that affects the data transmission of scatternets. Hence the Bluetooth devices follow broadcast mechanisms to re-build broken links which is called Link Recovery.

All available relay nodes information is stored in Relay connection table. Master constructs this table by using the information sent by the relay node. If any relay fails, master chooses the alternative relay using this table to make the transmission. For instance, relay r1 fails, recovery protocol construct the alternative path s11 $\rightarrow m 1 \rightarrow r 2 \rightarrow m 2 \rightarrow s 22$ by replacing $\mathrm{r} 1$ with $\mathrm{r} 2$. The procedure for link recovery is:

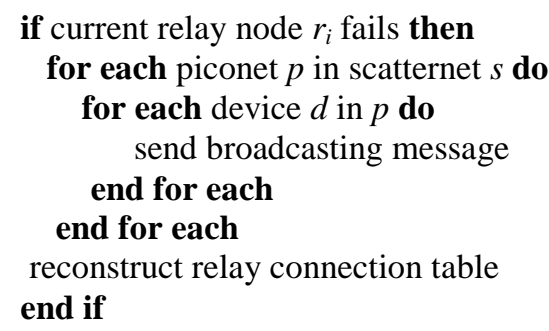

While performing with this protocol it is inferred that for small scale scatternet this practice performs very well while comparing to that of large scale scatternet. The main reason is that for large scale scatternet the time consumption is more, maintenance cost of relay node is high, extra resource utilization and higher consumption of AM_addr. The relay node maintenance cost is high since the broadcasting increases the control overheads, decrease the packet delivery rate and the network reliability in a bluetooth scatternet.

\subsection{Link Repairing}

Link Repairing Inter-piconet Communication technique overcomes the problems occurred by link breakage by repairing broken links [10]. Unlike the above method of broadcast route recovery, it repairs the links which are broken. If the master detects that the relay node is unreachable due to broken link, then it stars route repair process. In this fashion, primarily every master node is maintaining the information of relay 
nodes and it saves the relay ID, degree and clock offset. Then a repair message is sent, which is unicast, for repairing the broken links that are caused by relay node mobility. This scenario is illustrated as:

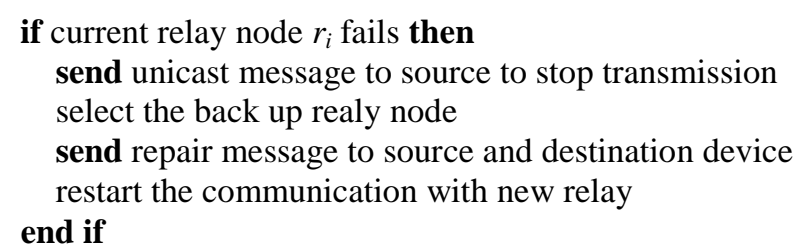

The message flooding is avoided since the repair messages are uni-cast and thus minimizes the control overhead. Due to the reason of utilizing optimum number of relays, it saves the energy for data communication in bluetooth scatternet and the throughput is high since the less possibility of packet loss.

\subsection{Link Breakage Prediction}

The process by predicting the possible link breakage before the link failure occurs is the method known as Link Breakage Prediction [11]. The prediction of link breakage is done based on the signal strength. A table is maintained by each master and bridge which will record all devices connected with it. An initial value is defined for the signal strength. When the value for master or relay node decreases then to avoid the link breakage, a new device will be selected as master or relay.

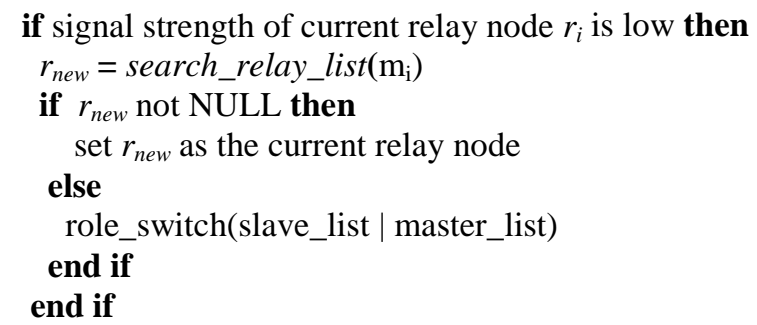

When the signal strength of master is going to decrease, then anyone of the slave will be selected to act as a master. In the case, when the relay node's link is going to break, then anyone of the relay node will be selected to act as relay node. In the above case, if there is no relay node, then role-switch operation will be performed. The procedure for breakage prediction is shown above.

\section{PERFORMANCE STUDY}

The performance of different scheduling protocols has to be re-assessed, considering the aforementioned metrics of Inter-piconet communication mechanism.The simulation is implemented using NS2 with Bluetooth extension. The performance of above mentioned metrics are analyzed with Round Robin algorithm to see whether or not its objective for efficient inter-piconet scheduling can be achieved. The simulation parameters are given in Table 1.

\subsection{Delay in network}

Link Breakage Recovery (LBR) uses broadcasting method to recover the broken links which is the time consuming process. Link repairing uses repair procedure to repair the link. Link Breakage Prediction (LBP) does not use any rebroadcast mechanism for finding the new route for broken links so it minimizes the total delay. Figure 2 shows the network delay for above mentioned metrics. As the total number of nodes in the scatternet increases, the network delay increases accordingly. Link Repairing and Link Breakage Prediction minimizes the network delay while transmitting the packets.

Table 1. Simulation parameters

\begin{tabular}{ll}
\hline Parameters & Value \\
\hline Number of nodes & $15-75$ \\
Number of nodes & $80 \mathrm{~m} \mathrm{x} 80 \mathrm{~m}$ \\
Number of nodes & $10 \mathrm{~m}$ \\
Traffic model & Constant bit rate (CBR) \\
Packet type & DH3, DH5 \\
Scheduling algorithm & RR \\
\hline
\end{tabular}

Indonesian J Elec Eng \& Comp Sci, Vol. 11, No. 2, August 2018 : 469 - 474 


\subsection{Throughput}

Throughput refers to the average rate of successful messages delivered or number of bytes received by a Bluetooth device per time unit. As the number of relays increase, there is also an increased probability of packet loss. During transmission link breakage affects the throughput of the scatternet as shown in Figure 3, LR is more effective than other metrics in terms of throughput.

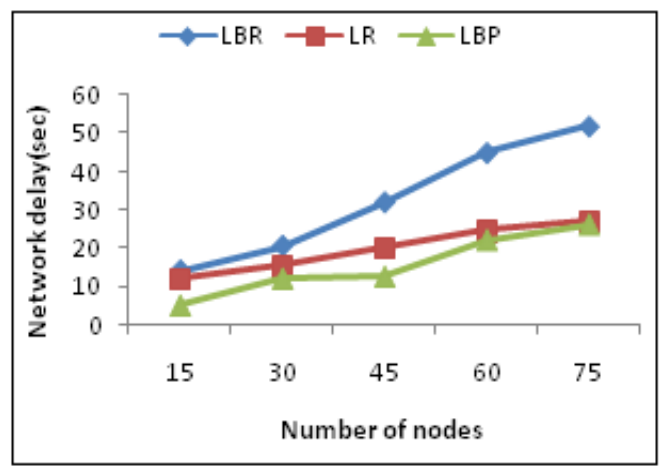

Figure 2. Network delay vs Number of nodes

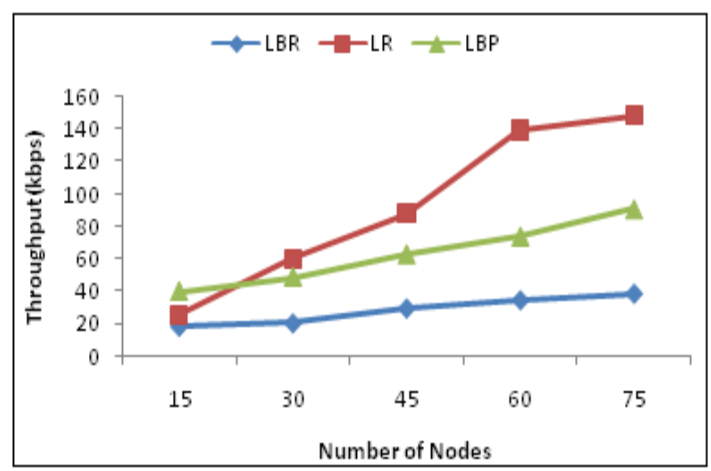

Figure 3. Throughput vs Number of nodes

\subsection{Packet Loss}

The failure of packets to arrive at their destination during transmission is known as packet loss. Figure 4 illustrates the average packet loss rate for different number of nodes. In LBR, failure of the link creates heavy packet loss. Link repairing and breakage prediction reduces no of packet loss during transmission.

\section{CONCLUSION}

In this paper, we have presented various performance metrics based on relay node to establish scheduling algorithm for a multi-hop wireless ad-hoc network based on Bluetooth technology. We also analyze the effect of considering these performance metrics on different number of nodes in terms of relay performance parameters. The inter-piconet scheduling in the scatternet topology has to consider these performance metrics to provide an efficient communication.

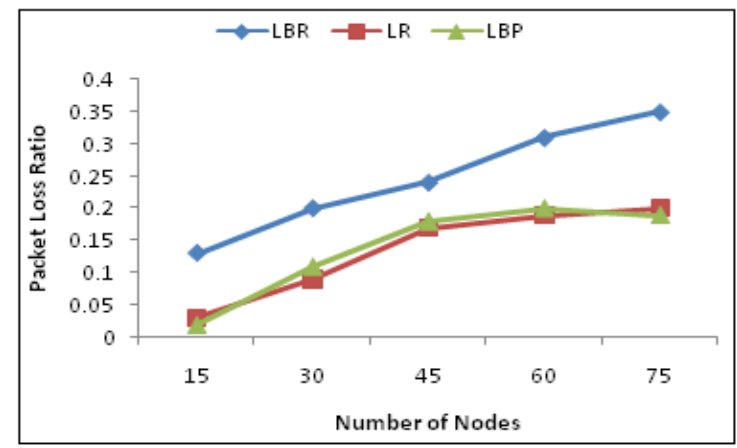

Figure 4. Packet Loss Ratio Vs Number of Nodes

\section{REFERENCES}

[1] Bluetooth Special Interest Group (SIG), std 2014, www.bluetooth.com

[2] JariValimaki, "Bluetooth and Ad Hoc Networking".

[3] VojislavB.Misic, JelenaMisic, “ Polling and Bridge Scheduling Algorithms in Bluetooth”, Technical report TR03/04, 2003. 
[4] LironHar-Shai, Ronen Kofman, Gil Zussmaan and Adrian Segall, "Inter-Piconet Scheduling in Bluetooth Scatternets”, Proc. OPNETWORK 2002, Aug. 2002.

[5] Manish Kalia, SumitGarg, Rajeev Shorey, "Scatternet Structure and Inter-Piconet Communication in the Bluetooth System”, IBM India Research Lab.

[6] Ramana Reddy, Et Al., "An Efficient Algorithm For Scheduling In Bluetooth Piconets And Scatternets," Wireless Networks, 2010,;16:pp. 1799-1816.

[7] Sheikh Tahir Bakhsh,Halabihasbullah, SabeenTahir, “ Dynamic Relay management protocol for efficient interpiconet scheduling in Bluetooth Scatternet”, Computers and Electrical Engineering, 2012; pp. 626- 642

[8] Johansson.P, Kapoor.R, Kazantzidis.M, Gerla.M,” Rendezvous Scheduling in Bluetooth Scatternets”, IEEE International Conference on Communications, 2002; pp.318-324.

[9] Chorng-Horng Yang, Chao-Wei Chou, Jiann-Horong Lin, Hsien-Lenig Tsai , “ Semi-Distributed Scatternet Reformation for improving Bluetooth Network Availability”, International Journal of Emerging Trends of Technology in Computer Science, 2014;3(3):pp.196-202.

[10] SabeenTahir, AbasMd Said, Sheikh TahirBakhsh,” Link Repairing for Inter-Piconet Communication Technique in Bluetooth Scatternet”, International Journal of Computer and Electrical Engineering,2012;4(4): pp.582-587.

[11] SabeenTahir,AbasMdSaid,"Route Breakage Prediction Protocol for Bluetooth Network Recovery”, Latest Advances in Information Science and Applications, 2012; pp.242- 247. 